\title{
Delivery of Multiple Child and Maternal Health Interventions during Supplementary Immunization Campaign in Rwanda, 2013: Lessons Learnt
}

\author{
Hassan Sibomana', Muhoza Jered ${ }^{2}$, Celse Rugambawa ${ }^{3}$, Jethro M. Chakauya ${ }^{4}$, Messeret E Shibeshi'* , Joseph \\ Okeibunor $^{5}$, Richard Mihigo ${ }^{5}$, Rajesh Bhaskar ${ }^{6}$ \\ 'Ministry of Health, Rwanda Biomedical Center, Rwanda \\ ${ }^{2} \mathrm{MOH}$ Rwanda \\ ${ }^{3}$ World Health Organization Country Office, Rwanda \\ ${ }^{4}$ World Health Organization Inter-Country Support Team, Harare, Zimbabwe \\ ${ }^{5} \mathrm{WHO}$ AFRO \\ Article Info \\ ${ }^{6}$ WHO Consultant, WHO Rwanda
}

Article Notes

Published: August 02, 2018

*Correspondence:

Dr. Messeret E Shibeshi, WHO Inter-Country Support Team,

East and Southern Africa, World Health Organization, 86

Enterprise Road, Highlands, Harare, Zimbabwe; Telephone

no: +47-241-38107; Email: eshetum@who.int

(c) 2018 Shibeshi EM. This article is distributed under the terms of the Creative Commons Attribution 4.0 International License.

\section{Keywords:}

Antigens

Campaign

Routine

Survey

Vaccination

Immunization

Vaccines

\section{ABSTRACT}

Objective: This paper assesses and describes the estimated coverage of the Measles Rubella (MR) campaign in each district; the national estimate of coverage for Human Papilloma Virus (HPV) vaccination campaign and Vitamin A supplementation simultaneously implemented in 2013.

Methods: We applied descriptive statistics and epidemiological tools to the outcomes of the campaigns to assess the coverage achieved on the different child and maternal health interventions. We also assessed the Adverse Events following Immunization (AEFI) where the evaluation was used at the same time to assess the routine immunization performance coverage for children 12-24 months for all childhood antigens, Tetanus Toxoid coverage among mothers of infants, combined with routine immunization performance evaluation, skilled delivery and bed nets use in Rwanda.

Results: Results indicated that among the eligible targets, 97.5\% received MR vaccine, $91 \%$ received HPV doses, and $83 \%$ got Vitamin $A$. The integrated vaccination of MR with HPV did not result in any serious AEFI. Coverage for antigens and doses given early in life was above $95 \%$ with card retention of $80 \%$. BCG to measles dropout by card was $8.5 \%$. Main reasons for nonvaccination indicated need for more specific immunization education. About $96.8 \%$ of mothers delivered in health institutions and $95 \%$ of the mothers slept under bed nets the night before the survey.

Conclusion: Rwanda successfully implemented an integrated coverage evaluation survey of the integrated vaccination campaign and routine immunization with statistically valid estimates. We drew lessons that information on routine immunization can be collected during post campaign survey evaluations. The district estimates should guide the programme performance improvement.

\section{Introduction}

Immunization programmes in the African Region have recorded remarkable successes in the last four decades, contributing to the eradication of smallpox, elimination of polio, control of measles and reduction by $99 \%$ of the incidence of other vaccine-preventable diseases $^{1}$. Similarly, tremendous progress has been made in the development of new vaccines, along with increasing access to new and underused vaccines in the lowest income countries.

The Rwanda the Ministry of Health provides routine vaccination coordinated through the national expanded programme on immunization. Between 1978 and April 2013 a total of 12 antigens 
had been introduced into the nation's immunization system. These include antigens such as Bacillus Calmettie Guuérin, Diphtheria, Pertussis, Tetanus, Oral Polio Vaccine, Measles, Hepatitis B, Haemophilus influenza type b, Pneumococcal Vaccine, Human Papilloma Virus, Rotavirus and Measles-Rubella antigens to prevent and control vaccine-preventable diseases. The impact of vaccination is reflected in the absence of any indigenous wild polio virus since 1993, achievement of the measles pre-elimination target and sustained maternal neonatal tetanus elimination since 2014. There has also been a consistent reduction in infant and child mortality in the country.

The programme has since developed a Measles Elimination Strategic Plan 2012-2020 in line with the WHO African Region technical guidance that aims to eliminate congenital rubella syndrome and introduce rubella-containing vaccine using the Measles Rubella (MR) formulation. It targets children 9 months -14 years to reduce the susceptible pool to be followed by introduction of MR vaccine into the routine immunization schedule. The introduction of the second dose of measles vaccine follows at 18 months of age with aim of achieving high performance coverage to prevent accumulation of susceptible and reach goal of elimination.

Advancing to reduce cancer of the cervix, Rwanda was among the first countries in the region to introduce the vaccine against Human Papilloma Virus (HPV) in 2011. At the time of the survey, a total of 2 cohorts of the target age group of school girls of 9-13 years had received three doses each giving to a total of six rounds of vaccination. During this campaign, taking advantage of the country's high school enrollment rates the vaccine was integrated with MR but delivered through schools targeting 12-15-yearold girls.

WHO recommends to Member States to conduct periodic assessments of immunization coverage using its standard survey guidelines to validate the reported coverage. Assessing coverage through high-quality surveys is an essential component of monitoring progress towards elimination, eradication or control of vaccine-preventable diseases ${ }^{2}$. Various methods to validate performance coverage including cluster surveys are routinely used in the immunization program ${ }^{3}$ and to assess reasons for nonvaccination and the success of communications strategies ${ }^{2}$. Rwanda thus conducted an evaluation of the integrated MR, HPV and Vitamin A supplementation campaign interventions through a coverage survey validation that was endorsed by the Inter-Agency Coordination Committee (ICC) to document lessons. The outcomes of the evaluation are presented in this paper.

\section{Methods}

The evaluation adopted the household population- based stratified two-stage cluster survey design. The study population consisted of children, who were eligible to receive MR (9-168 months) and Vitamin A (6-59 months) during the SIA. It also included girls aged 9-13 years eligible for previous doses and girls aged 12-15 years eligible for current dose of HPV. Children aged 12-23 months were evaluated of full routine immunization coverage, and mothers of infants aged 0-11 months for routine immunization with TT. Assessment data were collected in 2013, immediately after the campaign.

A total of 8563 Children aged 12-23 months; 11,941 women of reproductive age and 1366 Female adolescents were included in the sample. The WHO cluster sampling technique was used to select participants. Thirty (30) clusters in each of 30 districts were selected following probability proportional to size (PPS) method. Local enumeration areas were used to select clusters for the evaluation. The list of sectors used was provided by Central Statistics Office (CSO) to all teams with a list of clusters and enumeration areas surrounding clusters. Ten children were selected in each cluster by simple random sampling method. Nine hundred clusters were selected in the country targeting interviewing of 900 respondent mothers or caregivers.

The study aimed to get both national and district level estimates through the survey using immunization coverage standard questionnaire adapted from WHO Cluster Survey manual for all interventions, where applicable. Assessment of all the interventions was done at district level for both campaign and routine antigens with the exception of HPV where coverage was estimated nationally. The immunization coverage survey questionnaire captured respondents' socio-demographic information for target age groups, measles-rubella (age 9-168 months) and vitamin A (age 9-59 months), HPV received during SIA (girls 1215 years) for previous doses (girls 9-14 years ), routine vaccinations during the first 2 years of life (fully vaccinated defined as having received all antigens), Tetanus Toxoid (TT) vaccinations for women of childbearing age, place of delivery, use of bednets among infants and mothers the previous night from the survey, reasons for not receiving vaccinations, sources of information about SIA and SIA messages received.

Oral informed consent was obtained from parents or caretakers. The survey protocol was approved by the Scientific and Ethics Committee of the Ministry of Health of Rwanda.

All the survey teams were trained for 2 days that included practical field exercises and were supervised in the field. Questionnaires were checked and immediate feedback provided by phone calls. The 3 page-questionnaire took an estimated 15 minutes to complete for SIA data only 
and additional 10 minutes each for the routine child and TT sections. Immediately after field work ended, all teams were gathered for a half-day debriefing about experiences during field work and lessons learned.

\section{Statistical analysis}

All analyses were done in Epi Info using the sample survey procedures. The sampling structure took account of stratification, sample weighting for national estimates, and clustering. The calculation of the SIA and routine coverage rates was done using descriptive statistics. We also analyzed time between birth and routine vaccinations and the interval between doses with immunization cards to assess adherence to WHO recommended time intervals ${ }^{4,5}$.

Results

\section{Socio-demographic information}

Data were available on 8436 of the expected 8704 children for MR vaccine; 3939 of 4100 of children for vitamin A; 1366/1621 for HPV (girl child 12-14 years old); 8563/8773 for children 12-23 months for RI assessment $11,941 / 12,399$ mothers of infants $0-11$ months for TT. Eighty five percent of the respondents were from rural while $12 \%$ were from rural areas. The age group distribution for mothers indicated that 28\% were below 30 years; $42 \%$ were between $30-40$ years, and $30 \%$ were above 40 years. About $80 \%$ of the mothers had either primary or secondary education while only $20 \%$ were with no formal education. Agriculture was the main business of mothers and specifically 52\% were farmers while $13 \%$ daily laborers. Christianity is the dominant religion, Catholics, Protestants and Adventists together formed $>90 \%$ of respondents. Less than half (40\%) women had 1-2 children while $22 \%$ had $>3$ children, $32 \%$ had one child.

\section{Integrated Vaccination Campaign activities}

Overall the coverage was $97.5 \%$ for the MR vaccine, $83 \%$ for Vitamin A; 91\% for HPV dose achieved during integrated SIAs. Eighty-seven percent of the 8436 respondents had an immunization card. The crude coverage of MR vaccination was $97.5 \%$ as compared to $102 \%$ reported by the programme while the national coverage by card was only $84 \%$.

Card retention rate for Vitamin A for children aged 9 to59 months was $88 \%$ with coverage estimate of $83 \%$ while coverage validated by card alone was $75 \%$. The reported administrative coverage for Vitamin A (6-59months) was $86 \%$.

\section{Human Papillomavirus (HPV)}

The national HPV card retention rate was $87 \%$. The crude coverage of HPV during the campaign was $91 \%$ as compared to $99 \%$ reported administrative coverage. The non-response rates for HPV coverage started increasing sharply from HPV1 to HPV3. Ninety percent of girls receiving HPV were in school. The administrative coverage of inschool girls was $98.1 \%$ while for out of school was $1.1 \%$ indicative of high school enrollment in Rwanda among those surveyed. About half (53\%) of the respondents got their vaccination at school and $32 \%$ at health centers.

\section{Source of information on the SIAs}

Among the 8266 respondents, only $0.4 \%$ reported that they were not informed of the campaign before the implementation. The commonest sources of information on the campaign were radio and Community Health Workers (27\% each), followed by community leaders (25\%); school children in the family (13\%); and health workers in health centers $(8 \%)$.

\section{Childhood immunization}

The records showed immunization card retention rate was $80 \%$. The coverage for BCG was $99 \%$, and all antigens achieved more than $95 \%$ coverage. The rate for full immunization of children from card and recall combined was $94 \%$.

The reasons for non-immunization of children included lack of information and motivation among caretakers. Specifically, 18\% indicated that they were unaware of the time of vaccination, while $11 \%$ reported that they were not aware of the need for vaccination.

\section{Mothers of infants 0-11 months}

Eighty-seven percent received at least one injection of TT during the latest pregnancy. The proportion of women receiving two or more injections of TT, (TT2+) by card plus recall was $91.7 \%$ while only $18 \%$ from card only due to poor card retention (Table 1).

The protection at birth from tetanus in last pregnancy depending upon the number of TT injections received was 76\% from mothers' recall and card together. The Government health centers were the major source of providing TT vaccination (97.1\%).

Among 11,941 mothers of infants assessed, the card retention rates were only $36 \%$. The vaccination card retention rates were lower among new mothers despite the short time span of one year between child delivery and the evaluation survey.

\section{Reasons for failure for immunization among mothers}

There was a total of 990 inadequately vaccinated mothers where the main reasons cited included being unaware of the need for TT vaccination (25\%), and unaware of the need to return for another dose (35\%). Other reasons included postponed to another time (20\%), 
Sibomana H, Jered M, Rugambawa C, Chakauya JM, Shibeshi ME, Okeibunor J, Mihigo R, Bhaskar R. Delivery of Multiple Child and Maternal Health Interventions

Table 1: Results of 30 cluster RI evaluation survey-Rwanda 2013

\begin{tabular}{|c|c|c|c|c|c|c|c|c|c|}
\hline Total Respondents & & & & & 563 & & & & \\
\hline & & verage by & Card plus $\mathrm{Re}$ & & & Coverage & by Card On & & Admin Coverage* \\
\hline Indicators & $\%$ & Cl: Lower & Cl: Upper & DEFF & $\%$ & Cl: Lower & Cl: Upper & DEFF & \\
\hline Immunization cards available & $80 \%$ & $79 \%$ & $82 \%$ & 1.807 & & & & & \\
\hline BCG Scar present & $98 \%$ & $98 \%$ & $99 \%$ & 1.197 & & & & & \\
\hline BCG & $99 \%$ & $99 \%$ & $99 \%$ & 1.145 & $76 \%$ & $75 \%$ & $78 \%$ & 1.646 & $99 \%$ \\
\hline OPVO & $98 \%$ & $98 \%$ & $99 \%$ & 1.352 & $75 \%$ & $73 \%$ & $76 \%$ & 1.779 & \\
\hline OPV1 & $98 \%$ & $98 \%$ & $99 \%$ & 1.352 & $76 \%$ & $74 \%$ & $77 \%$ & 1.784 & \\
\hline OPV3 & $98 \%$ & $95 \%$ & $100 \%$ & 8.91 & $74 \%$ & $72 \%$ & $76 \%$ & 1.759 & $101 \%$ \\
\hline DTPHibHepB1 & $99 \%$ & $99 \%$ & $99 \%$ & 1.373 & $75 \%$ & $74 \%$ & $77 \%$ & 1.765 & $102 \%$ \\
\hline DTPHibHepB3 & $98 \%$ & $98 \%$ & $98 \%$ & 1.463 & $74 \%$ & $72 \%$ & $75 \%$ & 1.755 & $101 \%$ \\
\hline PCV1 & $98 \%$ & $98 \%$ & $99 \%$ & 1.481 & $75 \%$ & $73 \%$ & $76 \%$ & 1.76 & $102 \%$ \\
\hline PCV3 & $98 \%$ & $95 \%$ & $100 \%$ & 8.91 & $74 \%$ & $72 \%$ & $75 \%$ & 1.755 & $101 \%$ \\
\hline Measles & $97 \%$ & $96 \%$ & $97 \%$ & 1.435 & $71 \%$ & $67 \%$ & $75 \%$ & 3.754 & $102 \%$ \\
\hline Fully Immunized & $94 \%$ & $93 \%$ & $94 \%$ & 1.511 & $63 \%$ & $61 \%$ & $64 \%$ & 1.6568 & \\
\hline Fully Immunized by 1 year & $59 \%$ & $57 \%$ & $60 \%$ & 1.587 & & & & & \\
\hline OPV3 Valid doses & $76 \%$ & $74 \%$ & $77 \%$ & 1.227 & & & & & \\
\hline DTPHibHepB3 Valid Doses & $69 \%$ & $68 \%$ & $71 \%$ & 1.224 & & & & & \\
\hline PVC3 Valid Doses & $74 \%$ & $73 \%$ & $75 \%$ & 1.252 & & & & & \\
\hline Measles Valid Doses & $57 \%$ & $55 \%$ & $58 \%$ & 1.323 & & & & & \\
\hline
\end{tabular}

*Joint Reporting Form (JRF) 2012

lack of faith in immunization (10\%), time for vaccination not known (5\%) and being unaware of the place of immunization (5\%). Of the obstacles mentioned as a reason for not being vaccinated among the 990 respondents who were unvaccinated against TT were as follows: mother was too busy $31 \%$; family problems $23 \%$; child illness $15 \%$; TT vaccine not available $15 \%$; location of vaccination too far $8 \%$; and religious reasons $8 \%$.

\section{Antenatal care and deliveries}

The majority (87\%) of the women attended the health center for antenatal checkup at least once during the pregnancy. More than ninety-six percent (96.8\%) of the mothers delivered in health institutions. Thus, $97 \%$ of deliveries were conducted by skilled persons.

\section{Bed nets availability and use}

A high proportion of mothers (89.5\%) had bed nets and $95 \%$ of them slept under the nets the night before the survey. The bed-net distribution assessment indicated that $83.8 \%$ respondents received bed nets from the health centre during one of their visits for health services while $5.7 \%$ bought the bed nets from the market.

\section{Discussion}

Rwanda has been able to achieve conclusively its ambitious target of conducting a nationwide integrated post measles rubella and routine immunization coverage evaluation survey with district-wide statistically valid estimates. The country immunization programme has ably introduced MR vaccine into the system; the HPV vaccine has optimum coverage in the immunization programme as well as through integration with MR campaign.

The immunization card retention rates of $87 \%$ for MR campaign services allowed reliable estimation of the performance. The result confirmed that the target of coverage rate of $>95 \%$ for MR vaccination was achieved by crude coverage of $98 \%$ and $84 \%$ validated by card with confidence intervals between $1 \%$ and $3 \%$ indicating a high precision of estimates for both. For $96 \%$ of those who received MR dose during the campaign, it was their second opportunity to receive the measles vaccine indicating the accessibility of the services through routine immunization programme.

The national estimated coverage for HPV was 91\% and by card was $87 \%$. The vitamin A coverage for the country was $85 \%$ while the reported coverage for Vitamin A was $86 \%$. The optimum card retention for childhood immunization records documented at $80 \%$ may indicate awareness or value given by caretakers in keeping health records of their children. The routine immunization coverage for BCG was $99 \%$ and all the antigens achieved more than $95 \%$ coverage. The fully immunized child from card and recall was $94 \%$ that is above the minimum target of $90 \%$.

The dropout rate for BCG to measles by card and recall was $3.1 \%$ while by card only it was $8.5 \%$ which indicates fairly good service utilization. The reasons cut across all antigens and have implication for activities to sensitize and educate the people not only on the benefits of vaccination but also where and when they can get the vaccination. 
The role of such deliberately planned and implemented enlightenment activities for successful immunization was articulated in studies on vaccine refusal in Nigeria ${ }^{6-8}$.

The vaccination cards were also deficiently completed thus making monitoring of vaccination statuses difficult. It is evident that almost all the antigens registered a coverage rate of $>95 \%$ by card and recall together, however, all had lower coverage by card indicating a need to improve record keeping. The doses of routine immunization though given were not recorded on immunization card despite $80 \%$ children had cards at the time of the survey. This is a common challenge to tracking vaccination by conventional means ${ }^{9,10}$. Measles coverage by card and recall was $97 \%$ and $71 \%$ by card only. Valid dose for measles was $75 \%$. Almost all (94\%) children were found fully immunized with 11 basic antigens from card and history and only $78 \%$ fully immunized by card. It is notable that $73 \%$ of the children were fully immunized by the age of 1 year (from the card). Compared with administrative coverage of the country from Joint Reporting form and EPI coverage evaluation survey of 2007, the evaluation survey 2013 shows a clear and significant improvement in coverage of all indicators since 2007 and not too much deviation from administrative coverage.

The ANC coverage is fairly good with high health facility delivery. The card retention among young children (12-23mths) was $80 \%$. There is high coverage of bed net distribution and use for both mothers and children in Rwanda.

The TT profile revealed TT2+ coverage among pregnant women was $92 \%$. Protection at birth (PAB) was $76 \%$, while $98 \%$ deliveries took place either at health centre or the hospital and attended by skilled person. More than eighty-three percent (83.4\%) of pregnant women received bednets during their ANC visits. The results revealed a high utilization of health facilities both for delivery and antenatal care services. These results reflect a success story on the immunization programme in Rwanda.

Despite the success of the assessment, the exercise had a limitation. First is that the campaigns were not designed as studies so no robust analytical approach was built in from the planning stage. However, this did not affect the data as the reporting formats had provided publishable data in the past. The data were carefully cleaned and analyzed to provide necessary information used here. It also provided lessons upon which recommendations are made to improve on future activities. There is need to improve on record keeping. To do this, data collected during the campaigns should be transferred to the immunization cards to avoid failure in human memory.

\section{Conclusions}

Rwanda was able to implement an integrated coverage evaluation survey for the integrated MR, HPV vaccines inlcuding routine immunization surey. The evaluation provided valid estiments for both the campaign and routine immunization with district-wide statistically valid estimates.

In addition, the survey documented that no serious AEFI were reported by integrating MR with HPV vaccination during the campaign. The additional evaluation of bed net use and skilled delivery has demonstrated the successful integrated child and maternal health care delivery systems that exist in the health system in Rwanda.

The participation of district teams in data entry and data cleaning has extensively built the capacity of district teams. They should continue the practice of these skills for periodic assessment of program performance in their respective areas. The lesson from Rwanda should be extensively used for such elaborate coverage evaluation surveys.

\section{Sources of Financial Support}

This study was funded by the Ministry of Health Rwanda, WHO Rwanda and GAVI.

\section{References}

1. Larson HJ, Cooper LZ, Eskola J, et al. Addressing the vaccine confidence gap. Lancet. 2011; 378: 526-35.

2. World Health Organization. Global measles and rubella strategic plan: 2012-2020. Geneva, Switzerland 2012.

3. World Health Organization. Africa Regional Office. Measles SIAs Evaluation Guidelines. Brazzaville, Congo 2006.

4. Clark A, Sanderson C. Timing of children's vaccinations in 45 lowincome and middle-income countries: an analysis of survey data. Lancet. 2009; 373: 1543-1549.

5. World Health Organization. Measles vaccines: WHO position paper. Weekly epidemiological record No. 35, 2009; 84: 349-360.

6. Murele B, Vaz R, Gasasira A, et al. Vaccine perception among acceptors and non-acceptors in Sokoto State, Nigeria. Vaccine. 2014; 32(26): 3323-7.

7. Jegede AS. What led to the Nigerian boycott of the polio vaccination campaign. PLOS Medicine. 2007; 4: 417-422.

8. Renne E. Perspectives on polio and immunization in Northern Nigeria. Soc Sci Med. 2006; 63: 1857-1869.

9. Sanou A, Simboro S, Kouyaté B, et al. Assessment of factors associated with complete immunization coverage in children aged 12-23 months: a cross-sectional study in Nouna district, Burkina Faso. BMC International Health and Human Rights. 2009; 9(Suppl 1): S10.

10. De Wals P, De serres G, Niyonsenga T. Effectiveness of a mass immunization campaign against serogroup $\mathrm{C}$ meningococcal disease in Quebec. JAMA. 2001; 285: 177-181. 